\title{
The Effect of Fluoxetine on Sleep EEG in Childhood Depression: A Preliminary Report
} Roseanne Armitage, Ph.D., Graham Emslie, M.D., and Jeanne Rintelmann, B.A.

Fluoxetine is associated with substantial objective and subjective sleep disturbance in adults with major depressive disorders. In this preliminary report, the effects of fluoxetine on sleep electroencephologram (EEG) are described in 6 children and adolescents with nonpsychotic major depression. Fluoxetine increased light Stage 1 sleep, the number of arousals and rapid eye movement (REM) density. REM latency was largely unaffected. Oculomotor abnormalities were also evident on treatment, accompanied by an increase in myoclonic activity. Subjective sleep was also disturbed on treatment. These results are in keeping with those observed in depressed adults treated with fluoxetine. [Neuropsychopharmacology 17:241-245, 1997] (C) 1997 American College of Neuropsychopharmacology. Published by Elsevier Science Inc.
KEY WORDS: Depression; Sleep EEG; Fluoxetine; Children; Antidepressants

Several previous studies in adults with major depressive disorders (MDD) have shown that fluoxetine is associated with substantial objective and subjective sleep disturbance. Fluoxetine is a potent rapid eye movement (REM) sleep suppressor, decreasing total REM time and prolonging the latency to the first REM period, and is also associated with an increase in both light nonrapid eye movement (NREM) sleep and arousals (Cooper 1988; Hendrickse et al. 1994; Nicholson and Pascoe, 1988). Moreover, fluoxetine has been shown to induce oculomotor abnormalities in $30-60 \%$ of patients and is associated with increased incidence of nocturnal myoclonus and bruxism (Armitage et al. 1995; Dorsey et al. 1996; Keck et al. 1990, 1991; Schenck et al. 1992).

Fluoxetine and other selective serotonin reuptake inhibitors (SSRIs) are increasingly being prescribed to depressed children and adolescents as the first medicator,

From the University of Texas Southwestern Medical Center at Dallas, TX.

Address correspondence to: Roseanne Armitage, Ph.D., University of Texas Southwestern Medical Center, Department of Psychiatry, 5323 Harry Hines Boulevard, Dallas, Texas 75235-9070.

Received November 22, 1996; revised February 28, 1997; accepted March 21, 1997. primarily because of limited side effects, safety in overdose, and suggestion of efficacy (Ambrosini et al. 1995).

To our knowledge, no studies have explored the effects of fluoxetine on the sleep electroencephalogram (EEG) in depressed children and adolescents. This preliminary study reports the effects of fluoxetine on sleep EEG and oculomotor activity in 6 children and adolescents with major depression.

\section{METHODS}

\section{Subjects}

Six children and adolescents who met criteria for nonpsychotic major depression, but were generally medically healthy, participated in study. The average age of the sample of 4 girls and 2 boys was $12.0 \pm 1.9$ years. Participants were symptomatic and unmedicated for a minimum of 2 weeks prior to the baseline sleep assessment. Informed, written parental consent was obtained prior to study.

Diagnoses were based on the Diagnostic Interview for Children and Adolescents (DICA), a semi-structured DSM-III-R-Based diagnostic interview. Criterion depressive symptoms and severity were additionally assessed using the depressive items of the Kiddie Schedule for Af- 
fective Disorders and Schizophrenia (K-SADS) and the Children's Depression Rating Scale-Revised (CDRS-R).

Patients also completed two self-report scales for depression, either the Children's Depression Inventory (CDI) for those $\leqslant 12$ years of age or the Beck Depression Inventory for those $\geqslant 13$ years of age, and the Weinberg Screening Affective Scale (WSAS). Overall functioning was assessed with the Children's Global Assessment Scale (CGAS). Course of illness and the timing of prior and current episodes of depression was also established. The demographic and clinical information for participants at baseline and following several months of daily treatment with fluoxetine is shown in Table 1.

\section{Procedure}

Each participant spent two consecutive nights in the Sleep Study Unit at The University of Texas Southwestern Medical Center at Dallas baseline and again after several months of daily treatment with fluoxetine using methods previously described (Emslie et al. 1990, 1994). The average dose of fluoxetine was $18.3 \pm 4.1 \mathrm{mg}$ (range 10-20). Five of the 6 patients received $20 \mathrm{mg}$ daily; one patient received $10 \mathrm{mg}$ daily. The average length of time on treatment for the second sleep study was $10.9 \pm 4.6$ months (range 4.6-15.2). To rule out laboratory adaptation effects, data from the second night of study at each recording occasion are reported here.

Electroencephalographic data were collected from left (C3) and right (C4) central electrodes with a common reference (right and left ear lobes passed through a $10-\mathrm{K} \Omega$ resistor). Monopolar, left and right electrooculogram (EOG), and bipolar chin-cheek electromyogram (EMG) data were also recorded. A full electrode montage, used on the first night in the sleep laboratory, included leg, chest, and abdomen leads, and a nasal-oral thermistor to rule out independent sleep disorders. Leg leads were also used on the second night in the study. All electrophysiological signals were transduced by GRASS ${ }^{\mathrm{TM}}$ P-511 AC amplifiers and displayed on a paperless polygraph system. An amplifier sensitivity of 5 was used for EEG (50- $\mu \mathrm{V}, 0.5$-sec duration calibration), with half-amp

Table 1. Demographic and Clinical Characteristics of Outpatients with Major Depressive Disorder $(n=6)$

\begin{tabular}{lrrc}
\hline & Mean & SD & Range \\
\hline Females $\mathrm{n}=4$ & & & \\
Males $\mathrm{n}=2$ & & & \\
$\quad$ Age in years at evaluation & 12.0 & 1.9 & $10-14$ \\
$\quad$ Tanner stage & 2.8 & 1.2 & $1-4$ \\
$\quad$ Single episode $=4$ & & & \\
$\quad$ Recurrent episode $=2$ & & & \\
$\quad$ Length of current episode (in weeks) & 14.7 & 9.3 & $6-32$ \\
CDRS-R & & & \\
$\quad$ At baseline & 58.5 & 10.0 & $59-77$ \\
$\quad$ At second sleep & 24.8 & 7.4 & $17-2$ \\
\hline
\end{tabular}

low- and high-bandpass filters set at 0.3 and $30 \mathrm{~Hz}$, respectively. A $60-\mathrm{Hz}$ notch filter attenuated electrical noise. The EMG amplifiers were set at a sensitivity of 1 , with half-amp high filters at 30 and low filters set at 0.1 $\mathrm{Hz}$. Electrooculographic amplifiers were set at a sensitivity of 5 , with a half-amp high filters set at 30 and low filters set at $0.1 \mathrm{~Hz}$. Interelectrode impedances were maintained below $2 \mathrm{~K} \Omega$. Amplifiers were calibrated before and after each night's sleep. Data were digitized on-line through a 16-bit MICROSTAR ${ }^{\mathrm{TM}}$ analogue-to-digital (A/ D) board (EEG sampled at $250 \mathrm{~Hz}$ and EOG and EMG sampled at $62.5 \mathrm{~Hz}$ ) and stored on optical disk for offline analysis and stage scoring (Armitage et al. 1994).

Automated analyses were conducted on EOG and EMG activity in 30-seconds epochs. Similar EOG and EMG algorithms have been described elsewhere (Hoffmann et al. 1979). The algorithm computes the number of eye movements in each EOG channel larger or equal to $75 \mu \mathrm{V}$. An eye movement was defined as any positive or negative voltage excursions that hit threshold beyond baseline $(0 \mathrm{~V})$. The EOG threshold of $75 \mu \mathrm{V}$ was determined through repeated adjustments in sensitivity, until the detected events matched those that would be considered eye movements based on visual inspection. No duration criterion was applied to eye movement count (EOGC), nor were simultaneous eye movements required in the two EOG channels. The EOGC were then averaged for the right and left channels, producing an average EOG count per 30-seconds epoch. The EOG amplitude was computed as the total cumulative voltage of the EOG signal, with no minimum threshold. The EOG amplitude (EOGA) was then averaged for the right and left channels for each 30 -seconds epoch. The total cumulative voltage of the EMG signal defined EMG amplitude (EMGA) in each 30-seconds epoch, with no minimum threshold.

Pre- and post-sleep questionnaires were administered at both baseline and treatment, providing subjective estimates of sleep evaluations. Sleep quality, number of awakenings and how rested subjects felt upon awakening were queried.

Sleep EEG records were scored from C3 according to standard Rechtschaffen and Kales (1968) criteria by technicians trained at better than $90 \%$ agreement on an epoch-by-epoch basis. A number of sleep variables were derived from the sleep-stage score data. Total time in bed was computed as the total time from lights-out to wake-up time. Sleep onset latency was defined as the time elapsed before the first epoch of any sleep stage in a 10-minute segment containing no more than two minutes of wakefulness (Rush et al. 1989). Total sleep period (TSP) was defined as the length of time from sleep onset to wake up. Sleep efficiency was computed as the ratio of total sleep time to total time in bed $\times 100$. Rapid eye movement latency was equal to the number of minutes, including wake and movement time, from 
sleep onset to the first epoch of REM sleep (irrespective of REM period duration). Minutes and percentages of each sleep stage and of awake and movement time were computed in relation to TSP. Arousals were defined as the total number of awake episodes of $\geqslant 15 \mathrm{sec}-$ ond durations in TSP (Armitage et al. 1994).

For EOGC, EOGA, and EMGA, data were inspected visually to remove epochs with movement artifact or episodes of wakefulness ( $\geqslant 16 \mathrm{sec}$ ). The stage scores (REM, Stages 1, 2, 3, and 4) were then used to categorize EOG and EMG measures by sleep stage. Sleep records were also scored for the total number of myoclonic leg movements (i.e., electrical discharge) $>0.5$ seconds duration on each night in each sleep stage, but not in epochs scored as awake.

Statistical Procedures. Means and standard deviations were computed for EOGC, EOGA, and EMGA in each sleep stage, and data were coded by treatment level (baseline versus on-treatment). Separate repeated-measures analyses of variance (ANOVAs) were computed on EOGC, EOGA, and EMGA, and on standard sleep stage parameters. To minimize the likelihood of committing a Type I error, the minutes and \% of sleep stage were each treated as 5 variable multivariates (Stevens 1986). Univariate analyses were conducted on these variables only if a significant sleep stage main effect or interaction was obtained. All statistical analyses were conducted using SAS ${ }^{\mathrm{TM}}$ routines.

\section{RESULTS}

\section{Objective Sleep Parameters}

The means and standard deviations of objective sleep measures are shown in Table 2. Fluoxetine was associ-

Table 2. Means and Standard Deviations (in parentheses) of Sleep Parameters at Baseline and on Treatment $(n=6)$

\begin{tabular}{lcc}
\hline Parameter & Baseline & On Fluoxetine \\
\hline Time in bed & $510.0(45.4)$ & $517.3(43.1)$ \\
Total sleep period & $496.7(38.2)$ & $500.6(43.3)$ \\
Sleep latency & $14.3(15.2)$ & $17.7(10.2)$ \\
Sleep efficiency & $93.5(3.7)$ & $90.9(1.9)^{\mathrm{a}}$ \\
\% Stage 1 & $7.0(1.6)$ & $13.0(5.2)^{\mathrm{b}}$ \\
\% Stage 2 & $49.1(6.5)$ & $45.2(7.4)^{\mathrm{a}}$ \\
\% Stage 3 & $8.7(3.1)$ & $8.7(2.4)$ \\
$\%$ Stage 4 & $12.3(4.3)$ & $8.9(6.2)$ \\
\% REM & $18.9(3.5)$ & $18.4(1.7)$ \\
\% Awake & $4.0(1.9)$ & $5.9(2.3)$ \\
REM latency & $93.0(30.1)$ & $106.9(17.2)$ \\
REM density & $2.3(0.6)$ & $3.2(0.7)^{\mathrm{c}}$ \\
Arousals & $23.2(7.8)$ & $36.7(13.3)^{\mathrm{c}}$ \\
Myoclonic leg movements & $12.2(5.3)$ & $63.3(36.5)^{\mathrm{c}}$ \\
\hline
\end{tabular}

$$
\begin{aligned}
& { }^{\mathrm{a}} p<.10 . \\
& { }^{\mathrm{b}} p<.05 . \\
& { }^{\mathrm{c}} p<.02 .
\end{aligned}
$$

ated with significantly more Stage 1 compared to baseline $(\mathrm{F}=7.4, \mathrm{df}=1,4, \mathrm{p}<0.05)$. In fact, the amount of Stage 1 sleep nearly doubled on treatment. Fluoxetine also increased the total number of arousals during the night $(\mathrm{F}=15.6, \mathrm{df}=1,4, \mathrm{p}<0.02)$, and increased total REM density $(\mathrm{F}=12.3, \mathrm{df}=1,4, \mathrm{p}<0.02)$. Trends toward decreased sleep efficiency and \% Stage 2 sleep were also noted on treatment $(\mathrm{F}=4.94,4.7, \mathrm{df}=1,4, \mathrm{p}<$ 0.10 , respectively).

\section{Leg Movements}

The number of myoclonic leg movements also increased dramatically and significantly on fluoxetire $(F=11.9$,

\section{A: EOG AMPLITUDE}

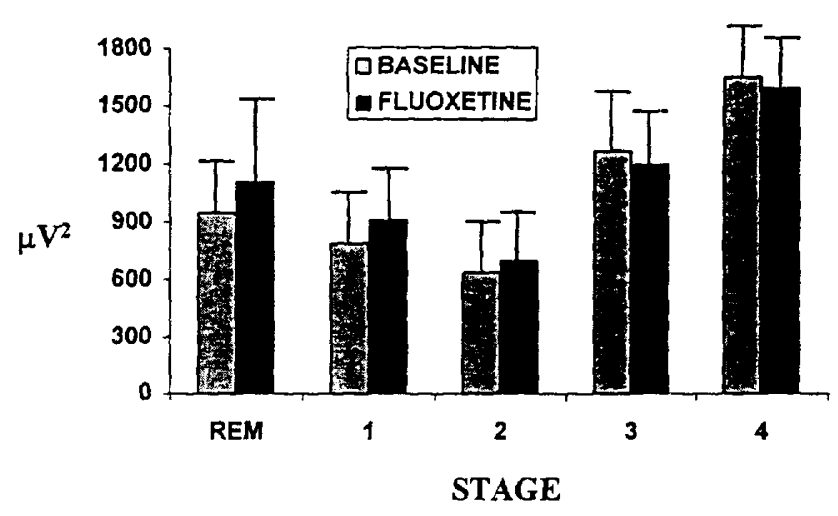

STAGE

\section{B: EMG AMPLITUDE}

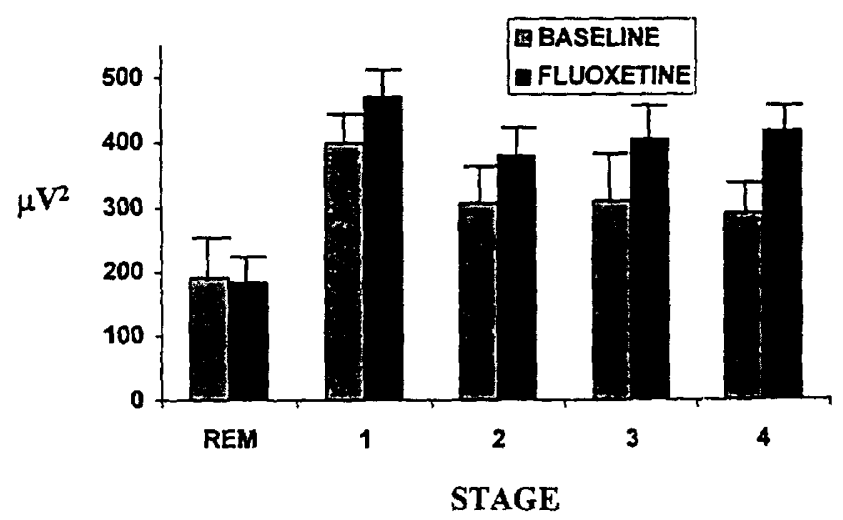

Figure 1. Amplitude (in $\mu \mathrm{V}^{2}$ ) of eye movements $\geqslant 75 \mu \mathrm{V}$ (A) and EMG activity (B) during each sleep stage at baseline (light bars) and during treatment (dark bars) with fluoxetine. Error bars denote standard deviations. 
$\mathrm{df}=1,4, \mathrm{p}<0.02$ ). In fact, there were five times as many leg movements on treatment (see Table 2), and increased leg movements were observed in all six patients.

\section{EOG and EMG Activity}

Significant treatment by sleep stage interactions were obtained for EOG $(\mathrm{F}=4.5, \mathrm{df}=4,16, \mathrm{p}<0.01)$ and EMG ( $\mathrm{F}=5.4, \mathrm{df}=4,16, \mathrm{p}<0.007)$ amplitude. Thus, fluoxetine had differential effects on EOG and EMG amplitudes across sleep stages. EMG amplitude increased in NREM, but not REM, whereas EOG amplitude was increased in REM and Stage 1 but decreased in Stages 3 and 4, accounting for the significant interaction. EOGC did not show significant treatment main effects or interactions. However, univariate analyses failed to reveal significant treatment main effects on the individual sleep stages for any of the measures. Trends were evident but did not reach significance, probably due to the small sample size and low statistical power.

The treatment-associated changes in EOG and EMG amplitude are shown in Figure 1 ( $\mathrm{A}$ and $\mathrm{B}$ respectively). Since the changes in EOGC virtually mirrored the amplitude measures, they are not shown. EMG amplitude was elevated on fluoxetine in all NREM sleep stages but not in REM, accounting for the treatment by sleep stage interaction. Increased EOG amplitude was most pronounced in REM and Stage 1 sleep. A slight decrease in EOG amplitude was found in Stages 3 and 4 sleep.

\section{Subjective Sleep Parameters}

With regard to subjective sleep parameters, 4 out of 6 patients rated sleep on fluoxetine as lower quality, with more awakenings and reported feeling less rested upon awakening. Five of the 6 patients also reported longer sleep latency on fluoxetine. ANOVA indicated trends toward treatment effects but none reached significance.

\section{DISCUSSION}

The preliminary study indicated that treatment with fluoxetine was associated with significantly more Stage 1 sleep, increased arousals, and increased REM density compared to baseline. REM sleep suppression was not evident on treatment. Oculomotor abnormalities were also present on treatment, as evidenced by sleep stage by treatment interaction for both EOG and EMG amplitude. Univariate analyses of oculomotor activity produced a significant increase in the number of leg movements. These effects are likely to be due to fluoxetine and not to clinical remission per se, since decreased Stage 1 sleep, REM density, and arousal would be expected in clinical remission. In addition, increased oculomotor activity appears to occur with selective serotonin reuptake inhibitors but not with other antidepressants, including those that also act on serotonin receptors (Armitage et al. 1994; Armitage, 1996). Moreover, the sleep architectural characteristic of this sample in remission continue to be significantly different (by posthoc $t$-test) from published data in normal controls (Emslie et al. 1990, 1994).

Some of these findings are in agreement with previous studies on the effects of fluoxetine in adults with MDD. However, others are in contrast to adult studies. With regard to objective sleep data in adults with MDD, fluoxetine has been shown to suppress REM sleep and increase both light NREM sleep and nocturnal wakefulness (Hendrickse et al. 1994; Nicholson and Pascoe 1988). The present study, confirmed increased Stage 1 sleep and arousals, but did not indicate significantly longer REM latency or decreased REM time in children treated with fluoxetine. The failure to find REM sleep suppression in children was unexpected.

Fluoxetine was also associated with increased oculomotor activity in children, but not in every sleep stage, as has been reported in adults (Armitage et al. 1995). More recently, Dorsey et al. (1996) reported fluoxetineinduced periodic limb movement disorder (PLMD) in $44 \%$ of adults with MDD. In children treated with fluoxetine, a significant increase in nocturnal myoclonic leg movements was obtained, although the present study used a very liberal criterion for leg movements. Increases on treatment were noted in all six patients. Moreover, using the same diagnostic criteria as Dorsey et al. (1992), 4 out of 6 children met criteria for a PLMD diagnosis on fluoxetine. None met criteria at baseline. It should be noted that many of the leg movements were intrusive and associated with subsequent arousal in the EEG, i.e., short duration bursts of alpha activity. Such "microarousals" will not be reflected in the arousal measure reported here since only awake episodes of $>15$ seconds were included. Thus, we have probably underestimated the alerting effects of fluoxetine. Several previous reports have suggested that antidepressant-induced nocturnal myoclonus is associated with excess serotonergic activity (Dorsey et al. 1996; Ware 1983). Others have suggested that the motoric abnormalities induced by fluoxetine are more likely to result from a reduction in dopamine rather than increased serotonin per se (Fishbain et al, 1992). We have suggested that fluoxetine-induced oculomotor abnormalities are likely to result from both primary and secondary serotonergic effects. Regardless of the mechanism of action, oculomotor abnormalities associated with fluoxetine appear in both children and adults with MDD.

These findings may have clinical significance. Children and adolescents do appear to notice the alerting effects of fluoxetine, in agreement with the previous re- 
ports in adults (Armitage et al. 1995; Dorsey et al. 1996). Most of the children (4 out of 6) reported lower quality, less-rested sleep with more awakenings on fluoxetine, in spite of the fact that all were in an episode of major depression at the initial sleep evaluation and were remitted during repeat sleep study. Persistent sleep disturbance may influence patient compliance and may necessitate augmentation with sedative compounds or hypnotics.

However, some caution should be exercised in interpreting the effects reported here, given the small sample size. Nevertheless, with the exception of REM suppression, fluoxetine appears to have an alerting effect on sleep in both children and adults with MDD.

\section{REFERENCES}

Ambrosini PJ, Emslie GJ, Greenhill LL, Kutcher S, Weller EB (1995): Selecting a sequence of antidepressants for treating depression in youth. J Child Adolescent Psychopharmacol 5:233-240

Armitage R, Rush AJ, Trivedi M, Cain J, Roffwarg HP (1994): The effects of nefazodone on sleep architecture in depression. Neuropsychopharmacology 10:123-127

Armitage R, Trivedi M, Rush AJ (1995): Fluoxetine and oculomotor activity during sleep in depressed patients. Neuropsychopharmacology 12(2):159-165

Armitage R (1996): Effects of antidepressant treatment on sleep EEG in depression. Journal of Psychopharmacology (Suppl 10) 1:22-25

Cooper GL (1988): The safety of fluoxetine-an update. Br J Psychiatry 153(3):77-86

Dorsey CM, Cunningham SL, Lukas SE, Winkelman JW, Richardson GS, Jones K (1992): Fluoxetine-induced eye movements during non-REM sleep. Sleep Res 21:55

Dorsey CM, Lukas SE, Cunningham SL (1996): Fluoxetineinduced sleep disturbance in depressed patients. Neuropsychopharmacology 14(6):437-442.

Emslie GJ, Rush AJ, Weinberg WA, Rintelmann JW, Roffwarg HP (1990): Children with major depression show reduced rapid eye movement latencies. Arch Gen Psychiatry 47:119-124
Emslie GJ, Rush AJ, Weinberg WA, Rintelmann JW, Roffwarg HP (1994): Sleep EEG features of adolescents with major depression. Biol Psychiatry 36:573-581.

Fishbain DA, Dominguez M, Goldberg M, Olsen E, Rosomoff H (1992): Dyskinesia associated with fluoxetine use. Case report. Neuropsychiatry, Neuropsychology, and Behavioral Neurology 5(2):97-100

Hendrickse WA, Roffwarg HP, Grannemann BD, Orsulak PJ, Armitage R, Cain JW, Battaglia J, Debus JR, Rush AJ (1994): The effects of fluoxetine on the polysomnogram of depressed outpatients: a pilot study. Neuropsychopharmacology 10(2):85-91

Hoffmann RF, Moffitt AR, Shearer JC, Sussman PS, Wells RB (1979) Conceptual and methodological considerations towards the developmental of computer-controlled research on the electro-physiology of sleep. Waking and Sleeping 3:1-16

Keck PE, Hudson JI, Dorsey CM, Matheson JK, Campbell PI (1990): Increased eye movements during non-REM sleep in fluoxetine-induced insomnia. Sleep Res 19:66

Keck PE Jr, Hudson JI, Dorsey CM, Campbell PI (1991): Effect of fluoxetine on sleep (letter). Biol Psychiatry 29:618-619.

Nicholson AN, Pascoe PA (1988): Studies on the modulation of the sleep-wakefulness continuum in man by fluoxetine, a 5-HT uptake inhibitor. Neuropsychopharmacology 27:597-602

Rechtschaffen A, Kales A (1968): A manual of standardized terminology, techniques and scoring system for sleep stages of human subjects. U.S. Government Printing Office, National Institute of Health Publication No. 204, Washington, DC.

Rush AJ, Giles DE, Jarrett RJ, Feldman-Koffler F, Debus JR, Weissenburger JE, Orsulak PJ, Roffwarg HP (1989): Reduced REM latency predicts response to tricyclic medication in depressed outpatients. Biol. Psychiatry 26:61-72.

Schenck CH, Mahowald MW, Kim SW, O'Connor KA, Hurwitz TD (1992): Prominent eye movements during NREM sleep and REM sleep behavior disorder associated with fluoxetine treatment of depression and obsessive-compulsive disorder. Sleep 15(3):226-235.

Stevens J (1986): Applied Multivariate Statistics for the Social Sciences. Hillsdale, Lawrence Erlbaum \& Associates.

Ware JC (1983): Tricyclic antidepressants in the treatment of insomnia. J Clin Psychiatry 463:(Pt2):59-67 